\title{
The microsurgical solution in supratentorial cavernous angiomas
}

\author{
A. SASARAN ${ }^{1}$, A. MOHAN ${ }^{2}$, H.A. MOISA ${ }^{3}$, A.V. CIUREA ${ }^{3,4}$ \\ ${ }^{1}$ Department of Neurosurgery, „Bagdasar-Arseni“ University Hospital, Bucharest, Romania \\ ${ }^{2}$ Bihor County University Hospital, Oradea, Romania \\ " "Carol Davila" University School of Medicine, Bucharest, Romania \\ ${ }^{4}$ Sanador Medical Center Hospital, Bucharest, Romania
}

\begin{abstract}
Cavernous angiomas of the central nervous system are rare, neurovascular lesions usually detected in patients aged 20-50 and represent up to 8-15\% of all intracranial malformations. The association between cavernomas and arteriovenous malformations is met in 10-30\% of cases.

Treatment strategies for intracranial cavernomas include conservative management, open microsurgery and finally stereotactic radiosurgery (the use of which is still highly debatable as there are no available methods to determine its short term effects).

The authors present their surgical experience in what regards a cohort of 149 consecutive patients, diagnosed with intracranial and supratentorial cavernomas, admitted, investigated and treated between January 2000 and January 2015. The clinical particularities of the patients together with the surgical approaches are debated. Last but not least the outcome and quality of life of the patients is discussed.
\end{abstract}

Keywords: intracranial cavernomas, management, neurosurgery, outcome, seizures

\section{INTRODUCTION}

Cavernous angiomas of the central nervous system are rare, neurovascular lesions usually detected in patients aged 20-50.

The first article in the literature, concerning an intracranial cavernoma, was published in 1854 by Hubert von Luschka (1) (1820-1875), who described a tumor-like vascular malformation, located in the brain of a patient.

Even though Bremer and Carson (2) were the first who managed to perform a surgical resection of an intracranial cavernoma, Walter Dandy (1886-1946) was the first to successfully publish a series of operated patients while taking note at the same time of the clinical features of these lesions. It was Dandy who noticed for the first time that intracranial cavernomas are predisposed to bleeding, epileptic seizures and focal neurological deficits. (3)

Intracranial cavernomas (IC) represent 0.02$0.53 \%$ of all intracranial lesions and $8-15 \%$ of all intracranial malformations. The association between cavernomas and arteriovenous malformations is met in $10-30 \%$ of cases. The lesions become symptomatic when the size is larger than $1 \mathrm{~cm}$. 
Treatment strategies for intracranial cavernomas include conservative management, open microsurgery and finally stereotactic radiosurgery (the use of which is still highly debatable as there are no available methods to determine its short term effects).

\section{MATERIAL AND METHOD}

The authors present their surgical experience in what regards a cohort of 149 consecutive patients, diagnosed with intracranial and supratentorial cavernomas, admitted, investigated and treated between January 2000 and January 2015.

The study includes 76 male patients and 73 female patients, all aged between 19 and 53 (y.o.). The mean age was 36.53 years in the general population. The mean age for male patients was 37.13 while the mean age for female patients was 35.9 years. (Fig. 1).

The collected data illustrates that 109 patients had single lesions while 40 patients had multiple lesions diagnosed. A number of 2 patients had histories significant for hereditary cavernomatosis, however, genetic analysis was not performed due to very high costs.

Neuroimagistic evaluation was performed using 1.5T Magnetic Resonance Imaging (MRI) on which the authors applied Zabramski's cavernoma classification scale (4) (Table 1). A pathology examination was performed for all cases, confirming the nature of the lesion.
TABLE 1. Cavernoma classification scale based on MRI aspects (after Zabramski et al.)

\begin{tabular}{|l|l|l|}
\hline $\begin{array}{l}\text { Lesion } \\
\text { type }\end{array}$ & MRI features & Pathology features \\
\hline I & $\begin{array}{l}\text { T1: hyperintense nidus } \\
\text { T2: hyper/hipointense } \\
\text { nidus }\end{array}$ & $\begin{array}{l}\text { Subacute } \\
\text { hemorrhage }\end{array}$ \\
\hline II & $\begin{array}{l}\text { T1: reticulate nidus with } \\
\text { mixed signal } \\
\text { T2: reticulate nidus with } \\
\text { mixed signal and } \\
\text { hipointense peripheral } \\
\text { rim }\end{array}$ & $\begin{array}{l}\text { Various foci of } \\
\text { thrombosis with } \\
\text { different ages }\end{array}$ \\
\hline III & $\begin{array}{l}\text { T1: iso/hipointense } \\
\text { nidus } \\
\text { T2: hipointense nidus } \\
\text { with hipointense } \\
\text { peripheral rim }\end{array}$ & $\begin{array}{l}\text { Chronic hemorrhage } \\
\text { with hemosiderin } \\
\text { impregnation } \\
\text { adjacent to the } \\
\text { lesion }\end{array}$ \\
\hline IV & $\begin{array}{l}\text { T1: invisible } \\
\text { T2: invisible }\end{array}$ & $\begin{array}{l}\text { Small cavernoma or } \\
\text { telangiectasia }\end{array}$ \\
\hline
\end{tabular}

In what regards the position of the lesions, the collected data (Table 2) shows that out of the 109 patients with single lesions a number of 39 patients had lesions in the frontal lobe (26.17\%); 14 patients $(9.39 \%)$ had lesions of the parietal lobe; 24 patients (16.1\%) had lesions of the temporal lobe; 8 patients had lesions of the occipital lobe (5.36\%); 8 patients had lesions of the basal ganglia (5.36\%); 4 cases were diagnosed with cavernomas within the sylvian fissure $(2.68 \%)$; 2 cases were diagnosed with cavernomas of the corpus callosum (1.34\%); 2 cases were diagnosed with cavernomas within the insula (1.34\%); a single case was positioned

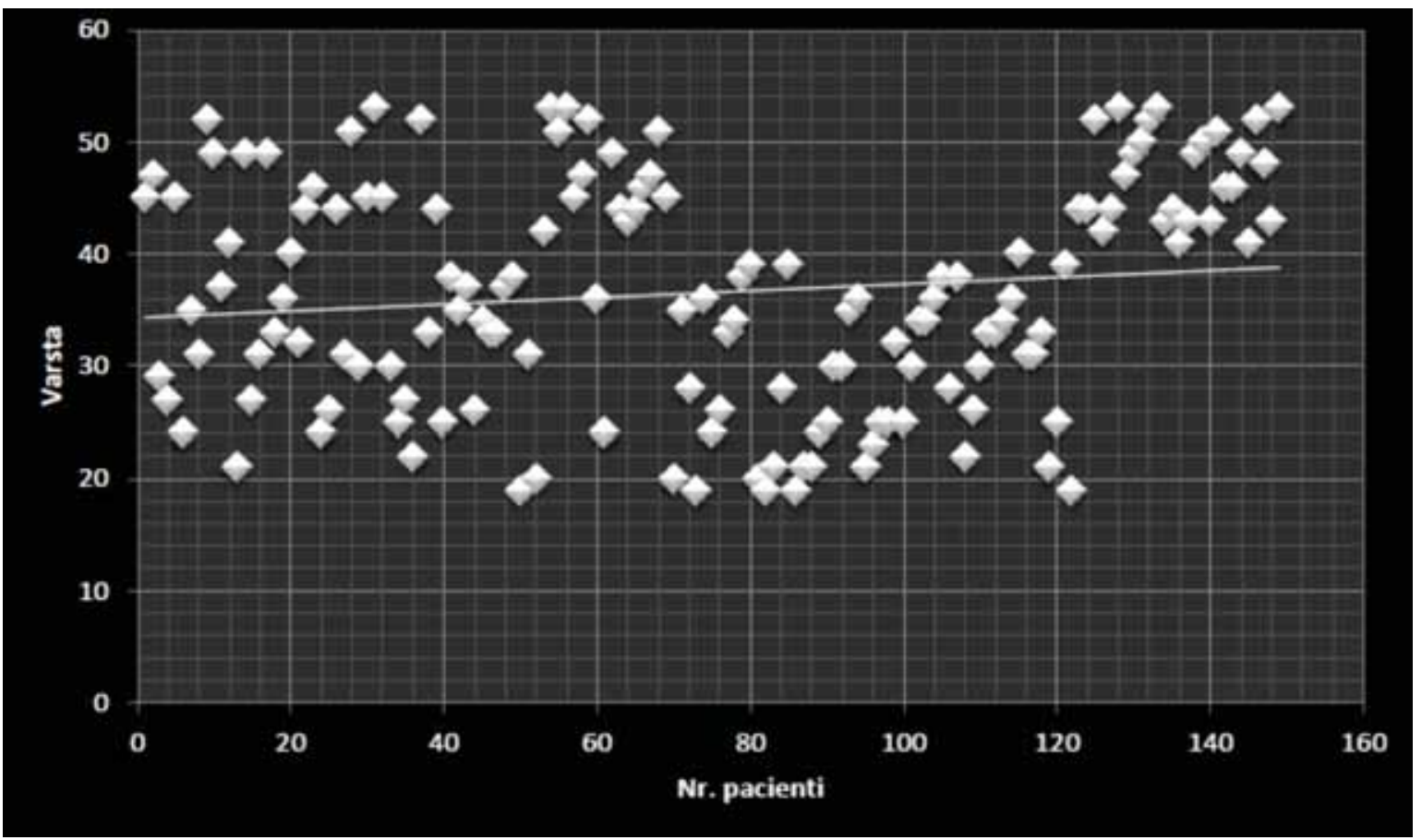

FIGURE 1. Dispersion chart illustrating the ages of the patients 
in a parasellar fashion $(0.67 \%) ; 5$ cases were diagnosed with intraventricular cavernomas (lesions within the lateral ventricles) (3.35\%); and 2 cavernomas were situated in the third ventricle $(1.34 \%)$.

TABLE 2. Position of lesions for single-lesion cavernomas in our study

\begin{tabular}{|l|c|c|}
\hline Position & Nr. of patients & $\mathbf{\%}$ \\
\hline Frontal & 39 & $26.17 \%$ \\
\hline Parietal & 14 & $9.39 \%$ \\
\hline Temporal & 24 & $16.10 \%$ \\
\hline Occipital & 8 & $5.36 \%$ \\
\hline Basal Ganglia & 8 & $5.36 \%$ \\
\hline Sylvian fissure & 4 & $2.68 \%$ \\
\hline Corpus callosum & 2 & $1.34 \%$ \\
\hline Insula & 2 & $1.34 \%$ \\
\hline Parasellar & 1 & $0.67 \%$ \\
\hline Lateral ventricle & 5 & $3.35 \%$ \\
\hline $3^{\text {rd }}$ ventricle & 2 & $1.34 \%$ \\
\hline Multiple lesions & 40 & $26.84 \%$ \\
\hline
\end{tabular}

\section{CLINICAL ONSET}

The clinical onset for the 149 cases evaluated in this study consisted of: Epileptic seizures for 55 patients (36.9\%), intracerebral hemorrhage in 55 patients (36.91\%), focal neurologic deficit (including cranial nerve palsy, sensitive and motor impairment) in 16 patients (10.73\%), memory loss in 2 patients (1.34\%), visual function impairment in 2 patients $(1.34 \%)$, disorientation in one patient $(0.67 \%)$, speech impairment in one patient $(0.67 \%)$ and hydrocephalus in 2 patients (1.34\%).

The patients manifested secondary epileptic seizures which were generalized tonic and clonic, simple and partial-complexe without any clinical correlation between the imaging of the lesions and the nature of the seizures. Headache was most of the times accompanied by nausea and vomiting and was considered to be the clinical translation of an intracerebral bleed. Visual function impairment was a consequence of cavernomas positioned within the occipital lobe or in the temporo-occipital region. In 3 patients with basal ganglia lesions hormonal imbalances were noticed and one such patient had a hyperkinetic disorder.

\section{NEUROIMAGING}

All the patients included in this study were subjected to neuroimagistic studies as a result of their symptoms. A procentage of $58 \%$ of the patients were subjected to initial Computed Tomography (CT) investigations, however, given the fact that CT scanning has low sensitivity and low specificity for intracranial cavernomas these patients were later subjected to MRI scanning which is the Gold Standard for intracranial cavernoma identification.

Given the fact that MRI imaging doesn't have the health risks associated with computed tomography it is the authors' opinion that MRI should become the first-intent imagistic technique for intracranial lesions.

On MRI scans intracranial cavernomas appear as heterogenous lesions, specifically described as having a „popcorn“-like aspect. A peripheral lowsignal rim is identified around the lesion. The peripheral low-signal rim is generated by macrophages rich in hemosiderin - a product generated by the decay of vascular hemoglobin.

Differential diagnosis is made with cerebral metastases, meningiomas, neurocysticercosis, or chronic granulomas.

\section{SURGICAL MANAGEMENT}

Microneurosurgery was the treatment method used for all the 149 patients included in this study. The indications for surgical interventions were represented by progressive neurosurgical symptoms and the existence of proof of bleeding.

The neurosurgical techniques used employed surgical microscopes, intraoperative neuronavigation and intraoperative ultrasound. Patients with hydrocephalus were first implanted with a ventriculo-peritoneal shunt system, to lower the pressure of the cerebrospinal fluid (CSF) and only afterwards was the initial lesion resected.

In our series 81 patients were considered surgical emergencies (54.3\%) and only 68 patients were electively operated after their symptoms stabilized. Surgical temporization depends on a series of factors which include the presence or absence of hemorrhage, the presence or absence of intractable epileptic seizures, the degree of compression generated by the lesion or the clinical onset. (5-8)

All surgical emergencies had major symptoms such as focal neurologic deficit (16 patients), hydrocephalus (2 patients) confirmed intracerebral hemorrhage (55 patients), progressive visual impairment ( 2 patients) and progressive headache with nausea and vomiting (6 patients). 
Essential for therapeutic success are MRI scanning and the use of adjuvant technologies such as electrophysiology and neuronavigation. Last but not least, modern neuroimaging such as functional MRI (fMRI) or intraoperative MRI (iMRI) as well as diffuse tensor imaging (DTI) are a wonderful add-on to the armamentarium of a neurosurgeon, however their cost was too great for them to be routinely employed.

Neuronavigation and intraoperative ultrasound are highly useful add-ons for a neurosurgeon in the operating theatre. They allow for minimal cortical trauma and the avoidance of vascular lesions or eloquent area damage enabling the neurosurgeon to perfectly control the surgical field even after brain collapse after large lesion resection. Such techniques were successfully used in 107 patients.

Surgical resection of supratentorial cavernomas is associated with a low morbidity and mortality risk. Given the fact that cavernomas are benign lesions the patients must be carefully selected to prevent quality of life drop. Local anatomy must be $100 \%$ respected.

In patients with multiple lesions literature certifies that only the symptomatic lesion must be resected. However, if the surgical corridor allows it, a second or even a third lesion can be removed (however only one surgical corridor may be constructed). In this respect, cortical mapping (Figure 2) has a central part as it allows to identify critical areas such as the center of speech. Electroencephalography (EEG) allows, if correctly performed, the identification of a clinically manifest lesion and its subsequent removal.

\section{SURGICAL PARTICULARITIES}

In patients with multiple lesions the target lesion is difficult to identify. For this reason, cor- tical mapping and EEG become mandatory. In patients with hereditary cavernomatosis there is a chance of de novo cavernomas appearing. Surgery is indicated for lesions generating masseffect and seizures via hemorrhage. (10-13)

Cavernomas in the orbit and affecting the optic nerve have a sudden onset with headache and visual impairment. The bleeding rate for such lesions is higher than $25 \%$ of all patients and intervention must be carried out urgently through a pterional or subfrontal approach. Such lesions must be removed rapidly as visual function deteriorates rapidly and permanently. Lesions can be positioned in the lateral portion of the intraconal space (prone for resection using a lateral orbitotomy). They can also be positioned inferiorly and can be approached using a transconjuctival intervention. They can affect the optic canal, the apex of the orbit and the superior orbital fissure.

Postoperatively visual function improves and the characteristic proptosis disappears, however, damage to the orbital fissure usually ends up with third nerve paralysis. $(5,6,14)$

Dural-based cavernomas are mostly positioned in and around the middle cerebral fossa. The clinical features of such lesions include visual deficit, hormonal imbalances and cranial nerve palsy. Unlike their intracerebral peers, dural cavernomas rarely bleed. On the other hand, dural cavernomas in the middle fossa have an intense vascularization and can bleed profusely intraoperatively. (15) For such lesions angiographies are recommended to be performed to allow for preoperative embolization. Literature data shows a morbidity and mortality higher than $40 \%$ for such lesions.

Capsular and thalamic cavernomas have a very high morbidity for open microsurgery. Usually the patients show significant postoperative

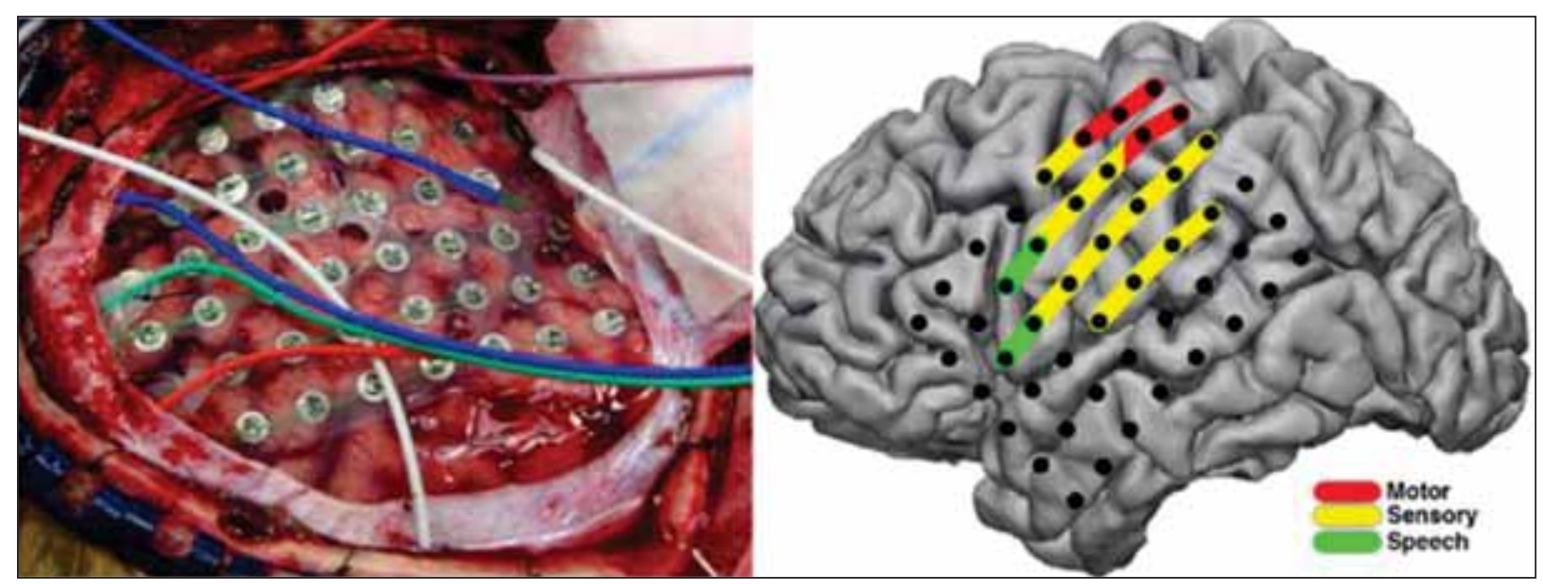

FIGURE 2. Cortical mapping of motor areas, sensory areas and speech areas (9) 
deficits and the resection for such cases is performed as much as a transcallosal approach allows it. (5)

Pineal area cavernomas have a heterogenous symptomatology with signs of intracranial hypertension, neurological symptoms, endocrine disfunction or visual function impairment. Lesions can be approached using a suboccipital and transtentorial approach, a supracerebellar and infratentorial approach or a posterior interhemispheric approach. Hydrocephalus, when identified can be treated using a ventriculoperitoneal shunt system.

Frontal lobe cavernomas are relatively easy to resect using a frontal craniotomy. Neuronavigation can be useful for centering the bone flap and for easy identification of the sagittal sinus thus avoiding vascular damage. Frontal parasagittal cavernomas can be approached using a bifrontal craniotomy which allows for easy retraction of the falxcerebri and therefore a widened exposure. Neuronavigation has the great advantage of being able to identify great vessels and therefore allows for their protection.

Parietal cavernomas can be resected with a standard craniotomy, again, assisted by neuronavigation. Deep and periventricular lesions can be approached either through the parietal lobe, either through a transcallosal approach which facilitates access to corpus callosum cavernomas, cingulate gyrus cavernomas and to parietal, occipital and mesial cavernomas. For lesions of the posterior area of the lateral ventricles the most appropriate approach is carried out through the superior part of the parietal lobe. The cortical incision must be carried out in a parallel fashion with the fibers of the superior parietal lobe, underneath the postcentral gyrus. (16)

Insular cavernomas can be approached using a dissection of the sylvian fissure, right above the lesion. The medial section of the fissure is not necessarily opened for posterior lesions. When the fissure is hard to delimitate a gentle subpial dissection can be performed. The dissection must be carefully carried out around the branches of the middle cerebral artery to avoid any complication and neuronavigation can prevent penetration of the internal capsule.(17)

Occipital cavernomas are harder to resect given the proximity of the visual cortex and optic fibers. The mesial part of the occipital lobe, along the superior and inferior poles of the calcarine fissure is situated one fissure above the lingual girus which lies on the tentorium. The further the lesion is situated from the occipital pole, the smaller the functional impact it will have. The occipital functional cortex can be easily identified using functional MRI. Stimulation of the visual field using luminescent diodes will increase the anatomical image. (17)

Cranial nerve cavernomas can affect the optic nerve, the oculomotor nerve, the facial / vestibulocochlear and hypoglossal nerve. Chiasmatic lesions require an orbito-zygomatic approach while cerebello-pontine angle lesions use a retrosigmoid approach. Based on the authors' experience cranial nerve cavernomas must be resected completely as symptoms appear early and subtotal resections usually end up with recurrences. (18)

\section{FOLLOW-UP}

The postoperative follow-up of all 149 patients was carried out using MRI studies at 2 months postop, 6 months postop, 1 year postop and 2 years postop. All the patients have a follow-up period of at least 36 months with a maximum range of 96 months.

\section{RESULTS}

Out of all 149 patients, per-primam total resections were achieved in 131 patients (87.9\%). 18 patients required a second intervention for complete resection of the targeted cavernoma.

Most of the patients had a favorable outcome scoring $4 p$ and $5 p$ on the Glasgouw Outcome Scale (GOS) (no disability, minimal symptoms, no impairment in daily activity). A number of 140 patients scored 5 on the GOS scale (93.9\%), 6 patients scored $4 p$ on the GOS $(4.02 \%)$ and only 3 patients scored $3 p$ on the GOS. (Table 3)

TABLE 3. Surgical results evaluated using the Glasgow Outcome Scale

\begin{tabular}{|c|c|c|}
\hline GOS & No. of patients & $\%$ \\
\hline 1 & 0 & 0 \\
\hline 2 & 0 & 0 \\
\hline 3 & 3 & $2.01 \%$ \\
\hline 4 & 6 & $4.02 \%$ \\
\hline 5 & 140 & $93.9 \%$ \\
\hline
\end{tabular}

A further detailed study of the outcomes using the Extended Glasgow Outcome Scale was carried out. The results which can be seen in Table 4 show Superior Good Recovery in 127 patients, Lower good recovery in 13 patients, Su- 
perior moderate disability in 6 patients, Lower moderate disability in 2 patients and superior severe disability in 1 patient.

TABLE 4. Clinical status of the patients

\begin{tabular}{|l|c|c|}
\hline Clinical Status & $\begin{array}{c}\text { No. of } \\
\text { patients }\end{array}$ & $\%$ \\
\hline Dead & 0 & 0 \\
\hline Vegetative state & 0 & 0 \\
\hline Lower severe disability & 0 & 0 \\
\hline Upper severe disability & 1 & $0.67 \%$ \\
\hline Lower moderate disability & 2 & $1.34 \%$ \\
\hline Upper moderate disability & 6 & $4.02 \%$ \\
\hline Lower good recovery & 13 & $8.72 \%$ \\
\hline Upper good recovery & 127 & $85.23 \%$ \\
\hline
\end{tabular}

Postoperative seizures were a result of the hemosiderin perilesional rim, which was a local iritative factor and the reactive gliosis. At 2 years since the intervention 35 patients $(23.48 \%)$ didn't manage to achieve seizure control. Despite a complete resection of eloquent area lesions, the gliotic ring was not completely removed. Even though in cases with multiple cavernomas the purpose was seizure control, in 17 cases $(11.4 \%)$ this was not achieved.

Postoperative deficits are a result of compression of eloquent areas and hemorrhage. They were observed in 25 patients. At 2 years since the intervention the number of patients that had compensated their deficits totaled 19. Unfortunately 6 patients had definitive deficits. A great number of cavernoma patients show minor or moderate deficits at admission. They are great candidates for surgery as recurrent hemorrhage increases the chance for deficits. Using adequate microsurgery techniques even eloquent area cavernomas can be successfully removed without any risk.
Postoperative infections were noticed in a single patient with a ventriculo-peritoneal shunt installed. In this patient the shunt system became infected and had to be replaced in a separate intervention. Last but not least, 60 patients showed psychological effects generated by the drop in quality of life.

\section{CONCLUSIONS}

Intracranial cavernomas are rare lesions, characterized by epilepsy in the majority of cases. When asymptomatic, the best management is clinical observation.

When symptomatology is dominated by seizures, the best prognosis results after the excision of the lesion and perilesional gliosis.

Neuronavigator-guided microsurgery achieved in $76.5 \%$ of all cases the removal of the lesions with good accuracy, thus providing a solution to the patients' seizures and improving the overall quality of life.

In what regards the 25 patients in which postoperative neurologic deficits were observed, 19 of them compensated the deficits and only 6 patients were left with definitive deficits. In this respect, the permanent deficits were a consequence of the pre-existing pathology of the patients.

Neuronavigation and adjuvant techniques such as cortical mapping, avoid the discomfort of the stereotactic frame. In patients with intracerebral hemorrhage, surgery is an emergency.

In multiple lesions, only the bleeding lesion must be operated.

The option of gamma-knife surgery in cavernomas is ineffective based on our experience and available literature data. $(19,20)$

\section{$\overline{\text { REFERENCES }}$}

1. Krayenbuhl H., Yasargil M.G. Die vaskulären Erkrankungenim Gebiet der Arteria vertebralis und Arteria basilaris. Stuttgart, Thieme-Verlag, 1957. pp 458-470.

2. Bremmer L., Carson N.B. A case of brain tumor (angiomacavernosum) causing spastic paralysis and attacks of tonic spasms: Operation. Am J Med Sci 100:219-242, 1890.

3. Dandy W.E. Venous abnormalities and angiomas of the brain. Arch Surg 17:715-793, 1928.
4. Zabramski J.M., Wascher T.M., Spetzler R.F., Johnson B., Golfinos J., Drayer B.P., Brown B., Rigamonti D., Brown G. The natural history of familial cavernous malformations: results of an ongoing study. J Neurosurg 80:422-432, 1994.

5. Ciurea A.V., Gambardella G. Actualități în cavernoamele intracraniene. Ed. Universitară „Carol Davila“, Bucureşti, 2005.

6. Kivelev J. Brain and Spinal Cavernomas - Helsinki Experience. University of Helsinki Press, 2010.
7. Bertalanffy H., Benes L., Miyazawa T., Alberti O., Siegel M., Sure U. Cerebral cavernomas in the adult. Review of the literature and analysis of 72 surgically treated patients. Neurosurg. Rev. 25(1-2): 1-53, 2002

8. Maraire J.N., Awad I.A. Intracranial cavernous malformations: Lesion behaviour and management strategies. Neurosurgery 37: 591-605, 1995.

9. Roland J., Hacker D., Breshears D., Gaona M., Hogan R., Burton H., Corbetta M., Leuthardt C. Brain mapping in a patient 
with congenital blindness - a case for multimodal approaches Front. Hum. Neurosci., 31 July 2013

10. Campeau N.G. Lane J.I. De Novo Development of a Lesion with the Appearance of a Cavernous malformation Adjacent to an Existing Developmental Venous Anomaly. Am J Neuroradiol 26:156-159, 2005

11. Detwiler P.W., Porter R.W., Zabramski J.M., Spetzler R.F. De novo formation of a central nervous system cavernous malformation: Implications for predicting risk of hemorrhage. Case report and review of the literature. J Neurosurg. 87: 629-632

12. Houtteville J.P. Brain cavernoma: A dynamic lesion. Surg Neurol 48:610-614, 1997

13. Tekkok I.H., Ventureyra E.C. De novo familial cavernous malformation presenting with hemorrhage 12.5 years after the initial hemorrhagic ictus: Natural history if an infantile form. Pediatr Neurosurg 25:151155, 1996.

14. Arrue P., Thorn-Kany M., Vally P. Cavernous Hemangioma of the intracranial optic pathways: CT and MRI. J Comput Assist Tomogr 23:357-361, 1999

15. Momoshima S., Shiga H., Yuasa Y., Kawase T. MR findings in extracerebral cavernous angiomas of the middle cranial fossa: Report of two cases and review of the literature. AJNR Am J Neuroradiol: 12:756-760, 1991

16. Lawton M., Golfinos J.G., Spetzler R.F. The contralateral transcallosal approach: experience with 32 patients. Neurosurgery 39:729-735, 1996.

17. Wanebo J.E., Lanzino G., Zabramski J.M., Spetzler R.F. Supratentorial cavernous malformations. In: Spetzler R.F. Cavernous Malformations. Operative techniques, Neurosurgery. 5(3):176-184, 2002

18. Deshmukh V.R., Albuquerque F.C., Zabramski J.M., Spetzler R.F. Surgical management of cavernous malformations involving the cranial nerves. Neurosurgery 53:352-357, 2003

19. Ciurea A.V. „Intracranial cavenromas: Personal experience of 109 cases and literature review", Cursul WFNS ClujNapoca, Romania, April, 2014

20. Steiner L., Karlsson B., Yen C.P., Torner J.C., Lindquist C., Schlesinger D. Radiosurgery in cavernous malformations: anatomy of a controversy. $J$ Neurosurg 113(1):16-21, 2010 